International Journal of Pure and Applied Mathematics

Volume 90 No. 2 2014, 143-152

ISSN: 1311-8080 (printed version); ISSN: 1314-3395 (on-line version)

url: http://www.ijpam.eu

doi: http://dx.doi.org/10.12732/ijpam.v90i2.4

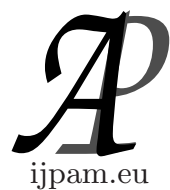

\title{
FIXED POINT THEOREMS FOR $\phi$-WEAKLY EXPANSIVE MAPPINGS IN METRIC SPACES
}

\author{
Shin Min Kang ${ }^{1}$, Manoj Kumar², Pankaj Kumar ${ }^{3}$, Sanjay Kumar ${ }^{4}$ \\ ${ }^{1}$ Department of Mathematics and RINS \\ Gyeongsang National University \\ Jinju, 660-701, KOREA \\ ${ }^{2}$ Department of Mathematics \\ Delhi Institute of Technology and Management \\ Gannaur, Sonipat 131001, India \\ Guru Jambheshwar University of Science and Technology \\ Hisar, 125001, INDIA \\ ${ }^{3}$ Department of Mathematics \\ Guru Jambheshwar University of Science and Technology \\ Hisar, 125001, INDIA \\ ${ }^{4}$ Department of Mathematics \\ Deenbandhu Chhotu Ram University of Science and Technology \\ Murthal, 131039, INDIA
}

\begin{abstract}
In this paper, we introduce the concept of $\phi$-weakly expansive mappings

AMS Subject Classification: 47H10, 54H 25

Key Words: $\phi$-weakly expansive mapping, weak reciprocal continuity, compatible mapping, $R$-weakly commuting mapping, $R$-weakly commuting mapping of type $\left(A_{f}\right)$, of type $\left(A_{g}\right)$ and of type $(P)$
\end{abstract}

Received: August 26, 2013

(c) 2014 Academic Publications, Ltd. url: www.acadpubl.eu

${ }^{\S}$ Correspondence author 


\section{Introduction}

In 1922, Banach proved a common fixed point theorem which ensures, under appropriate conditions, the existence and uniqueness of a fixed point. This result of Banach is known as Banachs fixed point theorem or Banach contraction principle. Many authors have extended, generalized and improved Banachs fixed point theorem in different ways.

Jungck [2] proved a common fixed point theorem for commuting maps, which generalized the Banach fixed point theorem. This theorem has had many applications but suffers from one drawback that the continuity of a mapping throughout the space is needed.

Definition 1.1. Let $f$ be a self-mapping of a metric space $(X, d)$. Then $f$ is said to be expansive if there exists a real number $h>1$ such that $d(f x, f y) \geq$ $h d(x, y)$ for all $x, y \in X$.

In 1997, Alber and Guerre-Delabriere [1] introduced the notion of $\phi$-weakly contraction as follows:

Definition 1.2. Let $f$ be a self-mapping of a metric space $(X, d)$. Then $f$ is said to be $\phi$-weakly contraction if there exists a continuous mapping $\phi$ : $[0, \infty) \rightarrow[0, \infty)$ with $\phi(0)=0$ and $\phi(t)<t$ for all $t>0$ such that $d(f x, f y) \leq$ $d(x, y)-\phi(d(x, y))$ for all $x, y \in X$.

In a similar mode, we introduce the notion of $\phi$-weakly expansive mappings in metric spaces as follows:

Definition 1.3. Let $f$ be a self-mapping of a metric space $(X, d)$. Then $f$ is said to be $\phi$-weakly expansive if there exists a continuous mapping $\phi$ : $[0, \infty) \rightarrow[0, \infty)$ with $\phi(0)=0$ and $\phi(t)>t$ for all $t>0$ such that $d(f x, f y) \geq$ $d(x, y)+\phi(d(x, y))$ for all $x, y \in X$.

Definition 1.4. Let $f$ and $g$ be two self-mappings of a metric space $(X, d)$. Then $f$ is said to be a $\phi$-weakly expansive with respect to $g: X \rightarrow X$ if there exists a continuous mapping $\phi:[0, \infty) \rightarrow[0, \infty)$ with $\phi(0)=0$ and $\phi(t)>t$ for all $t>0$ such that $d(f x, f y) \geq d(g x, g y)+\phi(d(g x, g y))$ for all $x, y \in X$.

In 1986, Jungck [3] introduced the notion of compatible mappings as follows:

Definition 1.5. Let $f$ and $g$ be two self-mappings of a metric space $(X, d)$. Then $f$ and $g$ are said to be compatible if $d\left(f g x_{n}, g f x_{n}\right)=0$, whenever $\left\{x_{n}\right\}$ is a sequence in $X$ such that $\lim _{n \rightarrow \infty} f x_{n}=\lim _{n \rightarrow \infty} g x_{n}=t$ for some $t \in X$.

An immediate consequence is that if $f$ and $g$ are compatible and $f z=g z$ ( $z$ is called a coincidence point of $f$ and $g$ ), then $f g z=g f z$. 
In 1994, Pant [6] introduced the notion of $R$-weak commutativity in metric spaces to extend the scope of the study of common fixed point theorems from the class of weakly commuting mappings to wider class of $R$-weakly commuting mappings as follows:

Definition 1.6. Let $f$ and $g$ be two self-mappings of a metric space $(X, d)$. Then $f$ and $g$ are called $R$-weakly commuting if there exists $R>0$ such that $d(f g x, g f x) \leq R d(f x, g x)$ for all $x \in X$.

Further, in 1997, Pathak et al. [10] improved the notion of $R$-weakly commuting mappings to $R$-weakly commuting mappings of type $\left(A_{f}\right)$ and of type $\left(A_{g}\right)$ as follows:

Definition 1.7. Let $f$ and $g$ be two self-mappings of a metric space $(X, d)$. Then $f$ and $g$ are called

(1) $R$-weakly commuting of type $\left(A_{g}\right)$ if there exists $R>0$ such that $d(f f x, g f x) \leq R d(f x, g x)$ for all $x \in X$.

(2) $R$-weakly commuting of type $\left(A_{f}\right)$ if there exists some $R>0$ such that $d(f g x, g g x) \leq R d(f x, g x)$ for all $x \in X$.

Definition 1.8. ([4])Let $f$ and $g$ be two self-mappings of a metric space $(X, d)$. Then $f$ and $g$ are called $R$-weakly commuting of type $(P)$ if there exists $R>0$ such that $d(f f x, g g x) \leq R d(f x, g x)$ for all $x \in X$.

In 1998 and 1999, Pant [7], [8] introduced a new notion of continuity, known as reciprocal continuity, as follows:

Definition 1.9. Let $f$ and $g$ be two self-mappings of a metric space $(X, d)$. Then $f$ and $g$ are called reciprocally continuous if $\lim _{n \rightarrow \infty} f g\left(x_{n}\right)=f t$ and $\lim _{n \rightarrow \infty} g f\left(x_{n}\right)=g t$, whenever $\left\{x_{n}\right\}$ is a sequence such that $\lim _{n \rightarrow \infty} f x_{n}=$ $\lim _{n \rightarrow \infty} g x_{n}=t$ for some $t \in X$.

If $f$ and $g$ are both continuous, then they are obviously reciprocally continuous, but the converse need not be true.

Recently, Pant et al. [9] generalized the notion of reciprocal continuity to weak reciprocal continuity as follows:

Definition 1.10. Let $f$ and $g$ be two self-mappings of a metric space $(X, d)$. Then $f$ and $g$ are called weakly reciprocally continuous if $\lim _{n \rightarrow \infty} f g x_{n}=$ $f t$ or $\lim _{n \rightarrow \infty} g f x_{n}=g t$, whenever $\left\{x_{n}\right\}$ is a sequence such that $\lim _{n \rightarrow \infty} f x_{n}=$ $\lim _{n \rightarrow \infty} g x_{n}=t$ for some $t \in X$.

If $f$ and $g$ are reciprocally continuous, then they are obviously weakly re- 
ciprocally continuous, but the converse need not be true.

In 2012, Manro and Kuman [5] proved the following fixed point theorem in complete metric spaces:

Theorem 1.11. Let $f$ and $g$ be two weakly reciprocally continuous selfmappings of a complete metric space $(X, d)$ satisfying

(C1) $g X \subset f X$;

$(C 2)$ there exists $q>1$ such that

$$
d(f x, f y) \geq q d(g x, g y)
$$

for all $x, y \in X$.

If $f$ and $g$ are either compatible or $R$-weakly commuting of type $\left(A_{g}\right)$ or $R$-weakly commuting of type $\left(A_{f}\right)$ or $R$-weakly commuting of type $(P)$, then $f$ and $g$ have a unique common fixed point.

In this paper, we prove common fixed point theorems for $\phi$-weakly expansive mappings, which generalize and extend the results of Manro and Kumam [5] using the concept of weak reciprocal continuity in metric spaces.

\section{Main Results}

Now, we prove main theorems using the notion of $\phi$-weakly expansive mapping.

Theorem 2.1. Let $f$ and $g$ be two weakly reciprocally continuous selfmappings of a complete metric space $(X, d)$ satisfying

(C1) $g X \subset f X$;

$(C 3)$ there exists a continuous mapping $\phi:[0, \infty) \rightarrow[0, \infty)$ with $\phi(0)=0$ and $\phi(t)>t$ for all $t>0$ such that

$$
d(f x, f y) \geq d(g x, g y)+\phi(d(g x, g y))
$$

for all $x, y \in X$.

If $f$ and $g$ are compatible, then $f$ and $g$ have a unique common fixed point.

Proof. Let $x_{0}$ be any point in $X$. Since $g X \subset f X$, there exists a sequence $\left\{x_{n}\right\}$ such that $g x_{n}=f x_{n+1}$. Define a sequence $\left\{y_{n}\right\}$ in $X$ by

$$
y_{n}=g x_{n}=f x_{n+1} .
$$

Moreover, we assume that if $y_{n}=y_{n+1}$ for some $n \in \mathbb{N}$, then there is nothing to prove. 
Now, we assume that $y_{n} \neq y_{n+1}$ for all $n \in \mathbb{N}$. From $(C 3)$, we have

$$
\begin{aligned}
d\left(y_{n}, y_{n-1}\right) & =d\left(f x_{n+1}, f x_{n}\right) \\
& \geq d\left(g x_{n+1}, g x_{n}\right)+\phi\left(d\left(g x_{n+1}, g x_{n}\right)\right) \\
& =d\left(y_{n+1}, y_{n}\right)+\phi\left(d\left(y_{n+1}, y_{n}\right)\right),
\end{aligned}
$$

that is,

$$
d\left(y_{n}, y_{n-1}\right)>d\left(y_{n+1}, y_{n}\right)
$$

Hence the sequence $\left\{d\left(y_{n+1}, y_{n}\right)\right\}$ is strictly decreasing and bounded below. Thus there exists $r \geq 0$ such that $\lim _{n \rightarrow \infty} d\left(y_{n+1}, y_{n}\right)=r$. Letting $n \rightarrow \infty$ in (2.2), we get $r \geq r+\phi(r)$, which is a contradiction, Hence we have $r=0$. Therefore

$$
\lim _{n \rightarrow \infty} d\left(y_{n+1}, y_{n}\right)=0 .
$$

Now, we will show that $\left\{y_{n}\right\}$ is a Cauchy sequence.

Let $\left\{y_{n}\right\}$ is not a Cauchy sequence. So there exists an $\epsilon>0$ and the subsequences $\left\{y_{m(k)}\right\}$ and $\left\{y_{n(k)}\right\}$ of $\left\{y_{n}\right\}$ such that minmal $n(k)$ in the sense that $n(k)>m(k)>k$ and $d\left(y_{m(k)}, y_{n(k)}\right)>\epsilon$. Therefore $d\left(y_{m(k)}, y_{n(k)-1}\right) \leq \epsilon$. By the triangular inequality, we have

$$
\begin{aligned}
\epsilon< & d\left(y_{m(k)}, y_{n(k)}\right) \\
\leq & d\left(y_{m(k)}, y_{m(k)-1}\right)+d\left(y_{m(k)-1}, y_{n(k)-1}\right)+d\left(y_{n(k)-1}, y_{n(k)}\right) \\
\leq & d\left(y_{m(k)}, y_{m(k)-1}\right)+d\left(y_{m(k)-1}, y_{m(k)}\right)+d\left(y_{m(k)}, y_{n(k)-1}\right) \\
& +d\left(y_{n(k)-1}, y_{n(k)}\right) \\
\leq & 2 d\left(y_{m(k)}, y_{m(k)-1}\right)+\epsilon+d\left(y_{n(k)-1}, y_{n(k)}\right) .
\end{aligned}
$$

Letting $k \rightarrow \infty$ in the above inequality and using (2.3), we get

$$
\lim _{k \rightarrow \infty} d\left(y_{m(k)}, y_{n(k)}\right)=\lim _{k \rightarrow \infty} d\left(y_{m(k)-1}, y_{n(k)-1}\right)=\epsilon .
$$

From $(C 3)$, we have

$$
\begin{aligned}
d\left(y_{m(k)-1}, y_{n(k)-1}\right) & =d\left(f x_{m(k)}, f x_{n(k)}\right) \\
& \geq d\left(g x_{m(k)}, g x_{n(k)}\right)+\phi\left(d\left(g x_{m(k)}, g x_{n(k)}\right)\right) \\
& =d\left(y_{m(k)}, y_{n(k)}\right)+\phi\left(d\left(y_{m(k)}, y_{n(k)}\right)\right) .
\end{aligned}
$$

Letting $k \rightarrow \infty$ and using (2.4), we get $\epsilon \geq \epsilon+\phi(\epsilon)$, which is a contradiction since $\phi(\epsilon)>\epsilon$. Hence $\left\{y_{n}\right\}$ is a Cauchy sequence in $X$. Since $X$ is complete, there exists a point $z \in X$ such that $\lim _{n \rightarrow \infty} y_{n}=z$. Therefore, by (2.1), we have

$$
\lim _{n \rightarrow \infty} y_{n}=\lim _{n \rightarrow \infty} g x_{n}=\lim _{n \rightarrow \infty} f x_{n+1}=z .
$$


Suppose that $f$ and $g$ are compatible mappings. Now, by the weak reciprocal continuity of $f$ and $g$, we obtain $\lim _{n \rightarrow \infty} f g x_{n}=f z$ or $\lim _{n \rightarrow \infty} g f x_{n}=g z$.

Let $\lim _{n \rightarrow \infty} f g x_{n}=f z$. Then the compatibility of $f$ and $g$ gives

$$
\lim _{n \rightarrow \infty} d\left(f g x_{n}, g f x_{n}\right)=0 .
$$

Hence $\lim _{n \rightarrow \infty} g f x_{n}=f z$.

Now, we claim that $f z=g z$. Let $f z \neq g z$. From (2.1), we get $\lim _{n \rightarrow \infty} g f x_{n+1}=$ $\lim _{n \rightarrow \infty} g g x_{n}=f z$. Therefore from $(C 3)$, we get

$$
d\left(f z, f g x_{n}\right) \geq d\left(g z, g g x_{n}\right)+\phi\left(d\left(g z, g g x_{n}\right)\right) .
$$

Letting $n \rightarrow \infty$, we get

$$
\begin{aligned}
d(f z, f z) & \geq d(g z, f z)+\phi(d(g z, f z)) \\
& >2 d(g z, f z),
\end{aligned}
$$

which is a contradiction. Hence $f z=g z$. Again the compatibility of $f$ and $g$ implies the commutativity at a coincidence point. Hence $g f z=f g z=f f z=$ ggz. Using (C3), we obtain

$$
\begin{aligned}
d(g z, g g z) & =d(f z, f g z) \\
& \geq d(g z, g g z)+\phi(d(g z, g g z)),
\end{aligned}
$$

which implies that $g z=g g z$. Also we get $g z=g g z=f g z$ and so $g z$ is the common fixed point of $f$ and $g$.

Next, suppose that $\lim _{n \rightarrow \infty} g f x_{n}=g z$. Since $g X \subset f X$, there exists $u \in X$ such that $g z=f u$ and therefore $\lim _{n \rightarrow \infty} g f x_{n}=f u$. The compatibility of $f$ and $g$ implies that $\lim _{n \rightarrow \infty} f g x_{n}=f u$.

Now, we claim that $f u=g u$. Let $f u \neq g u$. By virtue of (2.1), we have $\lim _{n \rightarrow \infty} g f x_{n+1}=\lim _{n \rightarrow \infty} g g x_{n}=f u$. From $(C 3)$, we have

$$
d\left(f u, f g x_{n}\right) \geq d\left(g u, g g x_{n}\right)+\phi\left(d\left(g u, g g x_{n}\right)\right) .
$$

Letting $n \rightarrow \infty$, we get

$$
d(f u, f u) \geq d(g u, f u)+\phi(d(g u, f u)),
$$

which is a contradiction. Hence $f u=g u$. Again the compatibility of $f$ and $g$ implies the commutativity at a coincidence point. Hence $g f u=f g u=f f u=$ ggu. Finally, using $(C 3)$, we obtain

$$
\begin{aligned}
d(g u, g g u) & =d(f u, f g u) \\
& \geq d(g u, g g u)+\phi(d(g u, g g u)),
\end{aligned}
$$


which implies that $g u=g g u$. Also, we get $g u=g g u=f g u$ and so $g u$ is the common fixed point of $f$ and $g$.

For the uniqueness, let $v$ and $w(v \neq w)$ be two common fixed points of $f$ and $g$. From $(C 3)$, we have

$$
\begin{aligned}
d(v, w) & =d(f v, f w) \\
& \geq d(g v, g w)+\phi(d(g v, g w)), \\
& =d(v, w)+\phi(d(v, w)),
\end{aligned}
$$

which implies that $v=w$. Hence $f$ and $g$ have a unique common fixed point. This completes the proof.

Next, we prove a common fixed point theorem for $R$-weakly commuting of type $\left(A_{g}\right)$, of type $\left(A_{f}\right)$ and of type $(P)$ as follows:

Theorem 2.2. Let $f$ and $g$ be two weakly reciprocally continuous selfmappings of a complete metric space $(X, d)$ satisfying $(C 1)$ and $(C 3)$. If $f$ and $g$ are $R$-weakly commuting of type $\left(A_{g}\right)$ or $R$-weakly commuting of type $\left(A_{f}\right)$ or $R$-weakly commuting of type $(P)$, then $f$ and $g$ have a unique common fixed point.

Proof. From Theorem 2.1, $\left\{y_{n}\right\}$ is a Cauchy sequence in $X$. Since $X$ is complete, there exists a point $z \in X$ such that $\lim _{n \rightarrow \infty} y_{n}=z$. Therefore, by (2.1), we have

$$
\lim _{n \rightarrow \infty} y_{n}=\lim _{n \rightarrow \infty} g x_{n}=\lim _{n \rightarrow \infty} f x_{n+1}=z .
$$

Now, suppose that $f$ and $g$ are $R$-weakly commuting of type $\left(A_{f}\right)$. The weak reciprocal continuity of $f$ and $g$ implies that $\lim _{n \rightarrow \infty} f g x_{n}=f z$ or $\lim _{n \rightarrow \infty} g f x_{n}=g z$.

Let $\lim _{n \rightarrow \infty} f g x_{n}=f z$. Then the $R$-weak commutativity of type $\left(A_{f}\right)$ of $f$ and $g$ yields, $d\left(g g x_{n}, f g x_{n}\right) \leq R d\left(f x_{n}, g x_{n}\right)$, and therefore $\lim _{n \rightarrow \infty} d\left(g g x_{n}, f z\right) \leq$ $R d(z, z)=0$, that is, $\lim _{n \rightarrow \infty} g g x_{n}=f z$.

Now, we claim that $f z=g z$. Let $f z \neq g z$. Again, using $(C 3)$, we get

$$
d\left(f z, f g x_{n}\right) \geq d\left(g z, g g x_{n}\right)+\phi\left(d\left(g z, g g x_{n}\right)\right) .
$$

Letting $n \rightarrow \infty$, we get

$$
d(f z, f z) \geq d(g z, f z)+\phi(d(g z, f z)),
$$


which is a contradiction. Thus, we get $g z=f z$. Again, by using the $R$-weak commutativity of type $\left(A_{f}\right)$, we have $d(g g z, f g z) \leq R d(g z, f z)=R d(z, z)=0$, that is, $g g z=f g z$. Therefore, $f f z=f g z=g f z=g g z$. Using (C3), we have

$$
\begin{aligned}
d(g z, g g z) & =d(f z, f g z) \\
& \geq d(g z, g g z)+\phi(d(g z, g g z)),
\end{aligned}
$$

which implies that $g z=g g z$. Then, we also get $g z=g g z=f g z$ and so, $g z$ is the common fixed point of $f$ and $g$.

Similarly, if $\lim _{n \rightarrow \infty} g f x_{n}=g z$, we can easily prove.

Suppose that $f$ and $g$ are $R$-weakly commuting of type $\left(A_{g}\right)$. Again, as done above, we can easily prove that $f z$ is a common fixed point of $f$ and $g$.

Finally, suppose that $f$ and $g$ are $R$-weakly commuting of type $(P)$. The weak reciprocal continuity of $f$ and $g$ implies that $\lim _{n \rightarrow \infty} f g x_{n}=f z$ or $\lim _{n \rightarrow \infty} g f x_{n}=$ $g z$.

Let $\lim _{n \rightarrow \infty} f g x_{n}=f z$. Then the $R$-weak commutativity of type $(P)$ of $f$ and $g$ yields $d\left(f f x_{n}, g g x_{n}\right) \leq R d\left(f x_{n}, g x_{n}\right)$, and therefore $\lim _{n \rightarrow \infty} d\left(f f x_{n}, g g x_{n}\right) \leq$ $R d(z, z)=0$, that is, $\lim _{n \rightarrow \infty} d\left(f f x_{n}, g g x_{n}\right)=0$. Using (2.1), we have, $f g x_{n-1}=$ $f f x_{n} \rightarrow f z$ and $g g x_{n} \rightarrow f z$ as $n \rightarrow \infty$.

Now, we claim that $f z=g z$. Let $f z \neq g z$. From $(C 3)$, we get

$$
d\left(f z, f g x_{n}\right) \geq d\left(g z, g g x_{n}\right)+\phi\left(d\left(g z, g g x_{n}\right)\right) .
$$

Letting $n \rightarrow \infty$, we get

$$
d(f z, f z) \geq d(g z, f z)+\phi(d(g z, f z)),
$$

which is a contradiction. Thus, we get $g z=f z$. Again, by using the $R$-weak commutativity of type $(P)$, we have $d(f f z, g g z) \leq R d(f z, g z)=0$, that is, $f f z=g g z$. Therefore, $f f z=f g z=g f z=g g z$. Using $(C 3)$, we have

$$
\begin{aligned}
d(g z, g g z) & =d(f z, f g z) \\
& \geq d(g z, g g z)+\phi(d(g z, g g z)),
\end{aligned}
$$

which implies that $g z=g g z$. Then we also get $g z=g g z=f g z$ and so $g z$ is the common fixed point of $f$ and $g$.

Similarly if $\lim _{n \rightarrow \infty} g f x_{n}=g z$, we can easily prove.

For the uniqueness, let $v$ and $w(v \neq w)$ be two common fixed points of $f$ and $g$. From $(C 3)$, we have

$$
\begin{aligned}
d(v, w) & =d(f v, f w) \\
& \geq d(g v, g w)+\phi(d(g v, g w)), \\
& =d(v, w)+\phi(d(v, w)),
\end{aligned}
$$


which implies that $v=w$. Hence $f$ and $g$ have a unique common fixed point. This completes the proof.

Remark 2.3. If we take $\phi(t)=q t$, where $q>0$, then Theorems 2.1 and 2.2 reduce to Theorem 1.11 .

Example 2.4. Let $X=[0,1]$ be equipped with the Euclidean metric $d(x, y)=|x-y|$ for all $x, y \in X$. Define $f, g: X \rightarrow X$ by $f x=16 x$ and $g x=4 x$. So, $g X=[0,4] \subset[0,16]=f X$.

Let $\left\{x_{n}\right\}$ be a sequence in $X$ such that $x_{n}=\frac{1}{n}$ for each $n$. Also, let $\phi$ : $[0, \infty) \rightarrow[0, \infty)$ be defined by $\phi(t)=2 t$ for all $t \in[0, \infty)$. Here, $f x_{n}=f \frac{1}{n}=\frac{16}{n}$. So $\lim _{n \rightarrow \infty} f x_{n}=0$. Also $\lim _{n \rightarrow \infty} f g x_{n}=\lim _{n \rightarrow \infty} f \frac{4}{n}=\lim _{n \rightarrow \infty} \frac{64}{n}=0=f 0$. So we can say that $f$ and $g$ are weakly reciprocally continuous. Also $d(f x, f y)=16|x-y|$, $d(g x, g y)=4|x-y|$ and $\phi(d(g x, g y))=8|x-y|$. Clearly

$$
d(f x, f y) \geq d(g x, g y)+\phi(d(g x, g y)) .
$$

Again $d\left(g g x_{n}, f g x_{n}\right)=d\left(g \frac{4}{n}, f \frac{4}{n}\right)=d\left(\frac{16}{n}, \frac{64}{n}\right)=\frac{48}{n}$ and $d\left(f x_{n}, g x_{n}\right)=d\left(\frac{16}{n}, \frac{4}{n}\right)=$ $\frac{12}{n}$. Clearly $d\left(g g x_{n}, f g x_{n}\right)<R d\left(f x_{n}, g x_{n}\right)$, where $R>4$. Hence $f$ and $g$ are $R$-weakly commuting mappings of type $\left(A_{f}\right)$. Also $f$ and $g$ are compatible. So all the conditions of Theorems 2.1 and 2.2 are satisfied and 0 is the unique fixed point of $f$ and $g$.

In Theorem 2.1 or Theorem 2.2, if $g$ is the identity mapping, then we obtain the following.

Theorem 2.5. Let $f$ be a surjective self-mapping of a complete metric space $(X, d)$ satisfying

$(C 4)$ there exists a continuous mapping $\phi:[0, \infty) \rightarrow[0, \infty)$ with $\phi(0)=0$ and $\phi(t)>t$ for all $t>0$ such that

$$
d(f x, f y) \geq d(x, y)+\phi(d(x, y))
$$

for all $x, y \in X$. Then $f$ has a unique fixed point.

Example 2.6. Let $X=\mathbb{R}$ and $d(x, y)=|x-y|$. Then $(X, d)$ is a complete metric space. Let $f x=4 x$ for all $x \in X$ and define $\phi:[0, \infty) \rightarrow[0, \infty)$ by $\phi(t)=2 t$. Clearly,

$$
\begin{aligned}
d(f x, f y) & =4|x-y| \\
& \geq 3|x-y| \\
& =d(x, y)+\phi(d(x, y)) .
\end{aligned}
$$

Also $f$ is surjective. So all the conditions of Theorems 2.5 are satisfied and zero is the unique fixed point of $f$. 


\section{Acknowledgments}

One of authors (Sanjay Kumar) is thankful to University Grants Comission for providing Major Research project under Ref. No. 39-41/2010(SR).

\section{References}

[1] Ya.I. Alber, S. Guerre-Delabriere, Principle of weakly contractive maps in Hilbert spaces, New results in operator theory and its applications, In: Oper. Theory Adv. Appl., vol 98, Birkhäuser, Switzerland (1997), 7-22, doi: 10.1007/978-3-0348-8910-0_2.

[2] G. Jungck, Commuting mappings and fixed points, Amer. Math. Monthly, 83, No. 4 (1976), 261-263, doi: 10.2307/2318216.

[3] G. Jungck, Compatible mappings and common fixed points, Int. J. Math. Math. Sci., 9, No. 4 (1986), 771-779, doi: 10.1155/S0161171286000935.

[4] S. Kumar, S.K. Garg, Expansion mappings theorems in metric spaces, Int. J. Contemp. Math. Sci., 4, No. 36 (2009), 1749-1758.

[5] S. Manro, P. Kumam, Common fixed point theorems for expansion mappings in various abstract spaces using the concept of weak reciprocal continuity, Fixed Point Theory Appl., 2012, No. 221 (2012), 12 pages, doi: 10.1186/1687-1812-2012-221.

[6] R.P. Pant, Common fixed points of non-commuting mappings, J. Math. Anal. Appl., 188, No. 2 (1994), 436-440, doi: 10.1006/jmaa.1994.1437.

[7] R.P. Pant, Common fixed points of four mappings, Bull. Calcutta Math. Soc., 90, No. 4 (1998), 281-286.

[8] R.P. Pant, A common fixed point theorem under a new condition, Indian J. Pure Appl. Math., 30, No. 2 (1999), 147-152.

[9] R.P. Pant, R.K. Bisht, D. Arora, Weak reciprocal continuity and fixed point theorems, Ann. Univ. Ferrara, 57, No. 1 (2011), 181-190, doi: 10.1007/s11565-011-0119-3.

[10] H.K. Pathak, Y.J. Cho, S.M. Kang, Remarks of $R$-weakly commuting mappings and common fixed point theorems, Bull. Korean Math. Soc., 34, No. 2 (1997), 247-257. 